\title{
SPEECH PERCEPTION OUTCOMES IN OLDER CHILDREN WHO USE MULTICHANNEL COCHLEAR IMPLANTS: OLDER IS NOT ALWAYS POORER
}

\author{
RICHARD C. DOWELL, PHD \\ ELIZABETH WINTON, MAUDSA
}

\author{
SHANI J. DETTMAN, MED \\ ELIZABETH J. BARKER, BAPPSC \\ MELBOURNE, AUSTRALIA
}

\author{
KATIE HILL, MAUDSA \\ GRAEME M. ClARK, MD, PHD
}

Speech perception outcomes for early-deafened children who undergo implantation as teenagers or young adults are generally reported to be poorer than results for young children. It is important to provide appropriate expectations when counseling adolescents and their families to help them make an informed choice regarding cochlear implant surgery. The considerable variation of results in this group makes this process more difficult. This study considered a number of factors in a group of 25 children who underwent implantation in Melbourne between the ages of 8 and 18 years. Each subject completed open-set speech perception testing with Bamford-Kowal-Bench sentences before and after implantation and preoperative language testing with the Peabody Picture Vocabulary Test. Data were collected regarding the type of hearing loss, age at implantation, age at hearing aid fitting, audiometric details, and preoperative and postoperative communication mode. Results were submitted to a stepwise multiple linear regression analysis with postoperative open-set sentence scores as the dependent variables. The analysis suggested that 3 factors have a significant predictive value for speech perception after implantation: preoperative open-set sentence score, duration of profound hearing loss, and equivalent language age. These 3 factors accounted for $66 \%$ of the variance in this group. The results of this study suggest that children who have useful speech perception before implantation, and higher age-equivalent scores on language measures, would be expected to do well with a cochlear implant. Consistent with other studies, a shorter duration of profound hearing loss is also advantageous. The mean sentence score for this group, $47 \%$, was not significantly different from the mean result across all children in the Melbourne program.

KEY WORDS - child, cochlear implant, predictive factor, speech perception.

\section{INTRODUCTION}

Many studies of the outcomes of cochlear implantation in children have identified the age of the child at the time of surgery as an important factor. ${ }^{1-7}$ It is generally accepted that younger children have an improved chance of speech perception, speech production, and language benefits from the application of implant technology. This rationale is often related to the concept of critical time periods for the development of spoken language, although the actual age intervals may vary for different skills and for different children. ${ }^{8}$ Despite acceptance that age may play a crucial role in the effective use of a cochlear implant, the wide range of results and other factors involved have sometimes tended to obscure this effect in clinical studies.

A number of recent studies have identified age at implantation or duration of profound deafness (which amounts to the same thing for congenitally deaf children) as having a significant relationship to speech perception outcomes. ${ }^{1-7}$ In general, children with congenital profound hearing impairment who undergo implantation as teenagers have not demonstrated the open-set speech perception abilities seen in many younger children who use cochlear implants. On the other hand, there have been cases of excellent speech perception results for older children. ${ }^{9}$ Notably, children with progressive hearing loss who undergo implantation at a stage when the hearing loss has reached a profound level often perform well on speech perception tasks. ${ }^{10}$ It has been suggested that the early use of residual hearing by such children may provide them with an advantage in processing auditory information from a cochlear implant.

The change in the clinical population that is considered for cochlear implantation may also be relevant to the effects of age on results. Because of improving outcomes, many children with a greater amount of residual hearing are being considered for implants. These children may have relatively good language and auditory processing skills in place, particularly as compared to children with profound-tototal congenital hearing loss. Their skills will depend not only on the level of residual hearing, but also on such factors as age of hearing aid fitting, level of family and educational support, and other individual attributes.

For the reasons outlined above, it may be time to

From the Department of Otolaryngology, University of Melbourne (Dowell, Clark), the Cooperative Research Centre for Cochlear Implant and Hearing Aid Innovation (Dowell, Dettman, Hill, Winton, Clark), the Bionic Ear Institute (Dowell, Clark), and the Cochlear Implant Clinic, Royal Victorian Eye and Ear Hospital (all authors), Melbourne, Australia.

CORRESPONDENCE - Richard C. Dowell, PhD, Dept of Otolaryngology, University of Melbourne Cochlear Implant Clinic, Royal Victorian Eye and Ear Hospital, 32 Gisborne St. East Melbourne, 3002, Victoria, Australia. 


\begin{tabular}{lrl}
\multicolumn{3}{c}{ SUBJECT DETAILS } \\
\hline Variable & Mean & \multicolumn{1}{c}{ Range } \\
\hline Age at implantation $(\mathrm{y})$ & 12.3 & $8.1-17.6$ \\
Age aided $(\mathrm{y})$ & 2.2 & $0.7-7.0$ \\
Duration of profound loss $(\mathrm{y})$ & 8.8 & $0.4-17.6$ \\
Experience with cochlear implant $(\mathrm{y})$ & 2.7 & $0.5-7.5$ \\
Pure tone average (best ear; $\mathrm{dB})$ & 112 & $82-130$
\end{tabular}

revisit the issues relating to the implantation of older children and attempt to identify some of the factors that could help in predicting outcomes. This paper will consider speech perception outcomes in particular, although it is accepted that the effects of implantation on language and speech production are of at least comparable importance.

\section{METHODS}

Subjects. The study considered the speech perception performance of 25 children who underwent implantation with the Nucleus multiple-channel cochlear prosthesis in Melbourne between 1988 and 1998. Their ages ranged from 8 to 18 years at the time of surgery. This sample represented $17 \%$ of the 145 children who underwent implantation during this time in the Melbourne program. The Table summarizes various demographic details of the subjects.

Data Collection. Speech perception was assessed for all 25 subjects with the Bamford-Kowal-Bench (BKB) sentence test ${ }^{11}$ presented in a free field at 70 $\mathrm{dB}$ sound pressure level with auditory input alone. Each list of BKB sentences comprises 16 sentences with 3 or 4 key scoring words per sentence. The score (out of 50) represents the number of key words correctly identified. Responses were either spoken or written by the subjects. When speech production could have affected the interpretation of responses, the test sessions were videorecorded and the responses were scored by 2 independent audiologists

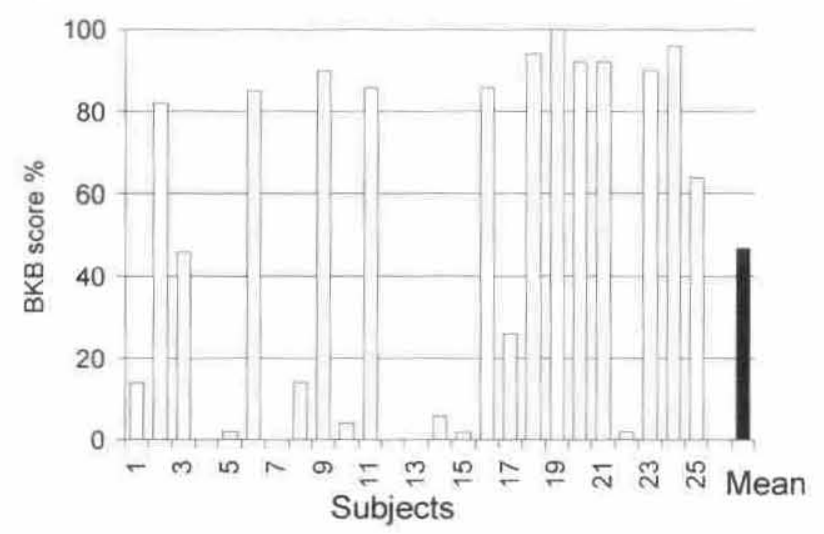

Fig 1. Postoperative scores for Bamford-Kowal-Bench (BKB) open-set sentences test for 25 older children with multiple-channel cochlear implants in audition-alone condition.

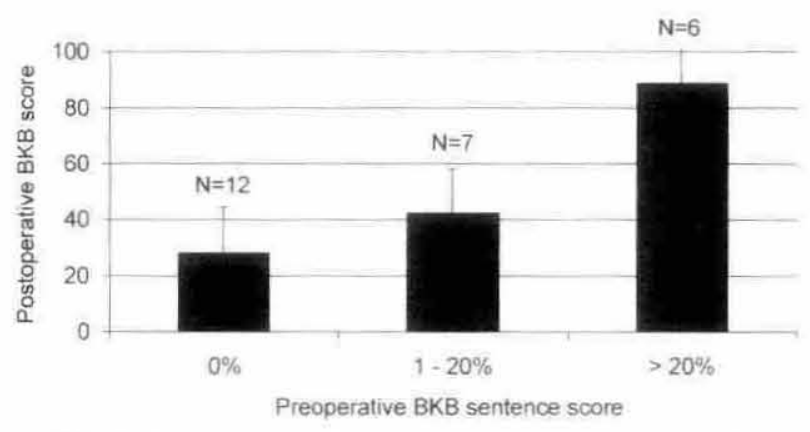

Fig 2. Postoperative BKB open-set sentence scores for 25 older children with multiple-channel cochlear implants, grouped according to preoperative scores.

or speech pathologists. Subjects who used hearing aids, as well as their cochlear implant, had their hearing aids switched off during testing. Most of the subjects had a number of postoperative assessments, but only the most recent assessment (until September 1999) was used in this study. All subjects had at least 12 months of experience with the cochlear implant system. All subjects used the spectral peak (SPEAK) speech processing strategy ${ }^{12}$ in a Spectra, Sprint, or Esprit processor (their take-home processor). The programming of the subjects' speech processors was checked with standard clinical techniques before speech perception testing. All subjects used at least 15 electrode combinations or channels in their speech processor MAPs.

In addition to the recent speech perception scores, data were obtained from the subjects' clinical files, including age at implantation, type of hearing loss (congenital profound, meningitis, progressive), age at hearing aid fitting, 3-frequency pure tone average hearing loss in the better ear at time of surgery, preimplantation speech perception scores for open-set $\mathrm{BKB}$ sentences with hearing aids, the duration of profound hearing loss before implantation, the preimplantation communication mode (exclusively oral or using some signing), and the preimplantation equivalent language age as estimated by the Peabody Picture Vocabulary Test (PPVT). ${ }^{13}$

Data Analysis. A stepwise linear regression analysis was carried out with the postoperative open-set sentence scores as the dependent variable and other information, as detailed above, as possible predictor variables. Because of the large number of predictor variables, a correlation analysis was used initially to indicate those predictor variables that were significantly correlated with the postoperative speech perception scores. Only these variables were used in the multiple regression analysis.

\section{RESULTS}

The open-set sentence scores for each of the subjects are shown in Fig 1 along with the overall mean 


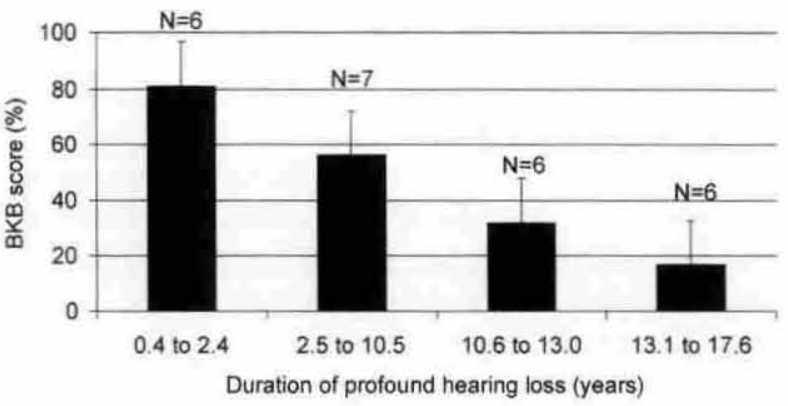

Fig 3. Postoperative BKB open-set sentence scores for 25 older children with multiple-channel cochlear implants, grouped according to duration of profound hearing loss before implantation.

for the group. Inspection of these data shows that there are approximately equal numbers of subjects who score poorly $(<10 \%)$ and subjects who score very well $(>80 \%)$, with very few scores in the middle range. This distribution leads to a rather misleading mean score of $47 \%$.

The stepwise multiple linear regression analysis suggested that 3 predictor variables accounted for significant amounts of variance in the postoperative BKB sentence scores. The open-set speech perception before implantation had a positive association with the results after implantation. This relationship is illustrated in Fig 2, in which the subjects are grouped according to their preimplantation speech perception scores.

The duration of profound hearing loss before implantation had a negative association with postoperative speech perception. This effect is illustrated in Fig 3, in which the subjects are grouped according to their duration of profound hearing loss.

The equivalent language (vocabulary) age as measured by the PPVT was positively associated with postoperative speech perception scores. This effect is illustrated in Fig 4, in which the subjects are grouped according to equivalent language age at the time of surgery. Note that Figs 2-4 do not necessarily provide an accurate description of the effect of the predictor variables, as interactions among these variables may enhance or decrease the apparent effects in a 2-dimensional representation. For instance,

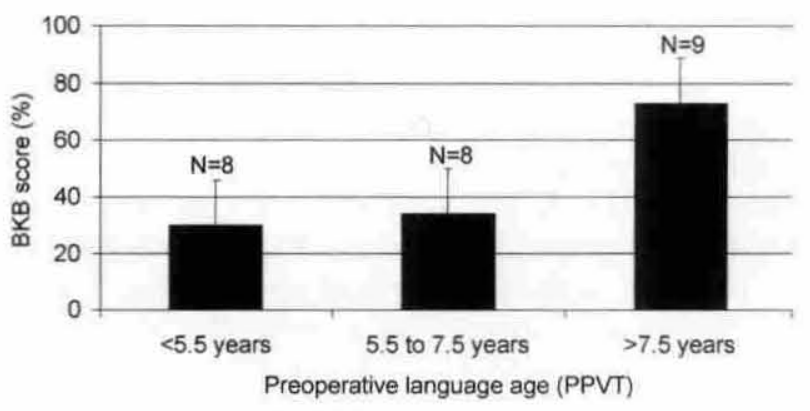

Fig 4. Postoperative BKB open-set sentences scores for 25 older children with multiple-channel cochlear implants, grouped according to preoperative equivalent language age. PPVT — Peabody Picture Vocabulary Test.

it is possible that subjects with better speech perception before implantation also have better language abilities. The correlations among the 3 significant predictor variables did not reach a significant level, but it is likely that such interactions do exist in reality. Despite these qualifications, the trends illustrated in the Figures correspond in direction with the results of the multiple regression analysis, and the analysis suggests that each of the variables has an independent effect on speech perception outcomes. The significant predictors accounted for $66 \%$ of the variance in the speech perception scores in this sample.

It is worth noting some of the variables that did not show any significant association with open-set speech perception. These included the hearing levels before implantation, as measured by the pure tone average in the better ear; the communication mode before implantation, with the subjects divided into those who used oral communication exclusively, and those who used some sign language; the type of hearing loss (congenital, meningitis, or progressive), and the age at hearing aid fitting. These variables have been identified as having significant associations with speech perception after implantation in previous studies of younger groups. In this study, however, these particular measures did not show significant effects on speech perception results.

\section{DISCUSSION}

Comparison With All Children With Implants. This

Fig 5. Distribution of BKB open-set sentence scores for all children who underwent implantation in Melbourne and who had completed this assessment.

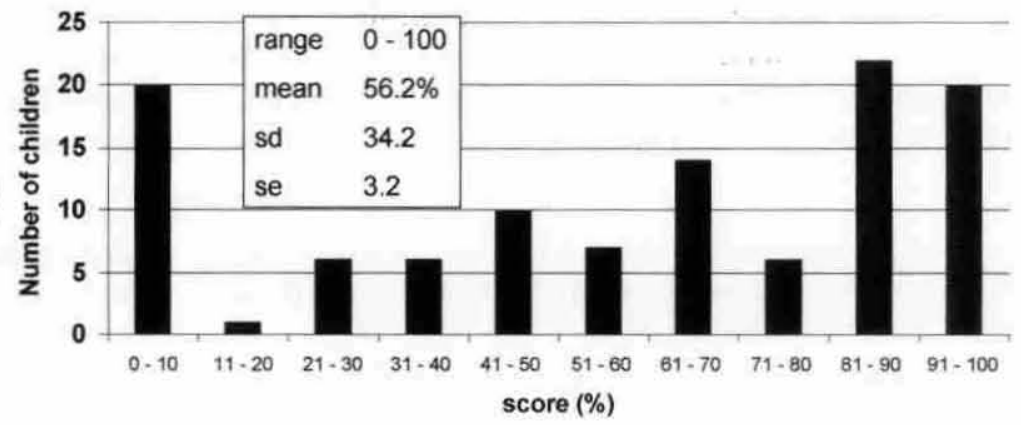


investigation of a small group of older children with cochlear implants shows that speech perception outcomes can be poor in some cases, but excellent results can be obtained for many others. The distribution of BKB sentence scores for all children in the Melbourne program is shown in Fig 5. Note that not all children with implants had reached the age at which this assessment was appropriate. The mean score was $56 \%$, which is not significantly different from the result for the group of older children. It does not appear to be valid to estimate the prognosis for a child cochlear implant candidate on the basis of age alone.

Preoperative Hearing and Speech Perception. The most significant effect found in this study was the predictive effect of preoperative speech perception scores on postoperative scores. Children who scored above $20 \%$ for BKB sentences beforeoperation tended to have speech perception scores close to $100 \%$ when using their implant system (Fig 2). On the other hand, the audiometric thresholds were not predictive of postoperative performance in this group. It seems that the ability to use minimal auditory information from hearing aids for speech perception carries over to effective use of the implant. This ability is likely to be affected by many aspects of the child's hearing loss, aid fitting, education, and cognitive abilities, and is not necessarily dependent on the preoperative audiogram alone.

Duration of Profound Hearing Loss. The duration of profound hearing loss has been identified in studies of both adults and children to be associated with speech perception results with cochlear implants. In this study, too, we see a link between this parameter and the sentence results (Fig 3 ). In the extreme, this result reflects the differences between a teenager who has a congenital profound or total hearing loss and a child of a similar age who becomes deaf suddenly after having normal hearing and education for their early life. Between these extremes are children who may have had some progression in hearing loss over time such that their early opportunities for auditory learning were more favorable than those of others. The result supports the hypothesis that auditory deprivation can lead to detrimental effects on speech perception performance. It also is consistent with the idea that the development of language through audition in early life will enhance the later use of audi- tory information from cochlear implants.

Language Development. Vocabulary development as measured by the PPVT was related to speech perception outcomes in this study. Because the speech perception test used involved connected sentences, various aspects of language knowledge are bound to come into play in completing the task. These include both semantic and syntactic knowledge. Figure 4 indicates that children with equivalent language ages below 7.5 years tend to struggle with this sentence perception task. The result probably also reflects a general relationship between language development and speech perception ability, as demonstrated by Blamey et $\mathrm{al}^{14}$ on a larger group of hearing-impaired children. Other studies have identified the mode of communication for children with cochlear implants as having a significant relationship with speech perception abilities, ${ }^{1.10}$ but this study did not indicate that the communication mode was a relevant factor for these older children. Vocabulary development obtained through the use of oral or sign language communication appeared to be sufficient to assist speech perception.

\section{CONCLUSIONS}

The results of this investigation suggest that for older children considering cochlear implantation, it is important to assess language development and the current use of residual hearing for speech perception, and to have a detailed history of the progression of the hearing loss. These factors can have significant effects on speech perception outcomes, and the information may help to provide more useful counseling to candidates and their families. The estimation of outcomes in the preimplantation phase for this group is particularly important, as results do vary over an extreme range.

This study provides support for the widening of indications for cochlear implantation, in terms of degree of hearing loss. On the other hand, the consideration of children with useful residual hearing brings with it an obligation to assess perceptual performance with hearing aids in a rigorous manner. In addition to standard testing, an evaluation that considers each ear separately and considers the binaural advantage for speech perception in both quiet and background noise appears necessary for children with useful aided hearing in both ears.

\section{REFERENCES}

1. Dowell RC, Blamey PJ, Clark GM. Potential and limitations of cochlear implants in children. Ann Otol Rhinol Laryngol Suppl 1995;104(suppl 166):324-7.

2. Fryauf-Bertschy H, Tyler RS, Kelsay DM, Gantz BJ. Woodworth GG. Cochlear implant use by prelingually deaf- ened children: the influence of age at implant and length of device use. J Speech Lang Hear Res 1997:40:183-99.

3. Hodges AV, Dolan Ash M, Balkany TJ, Schloffman JJ, Butts SL. Speech perception results in children with cochlear implants: contributing factors. Otolaryngol Head Neck Surg 
1999;121:31-4.

4. Meyer TA, Svirsky MA, Kirk KI, Miyamoto RT. Improvements in speech perception by children with profound prelingual hearing loss: effects of device, communication mode. and chronological age. J Speech Lang Hear Res 1998;41:84658.

5. Nikolopoulos TP, O'Donoghue GM, Archbold S. Age at implantation: its importance in paediatric cochlear implantation. Laryngoscope 1999;109:595-9.

6. Snik AFM, Makhdoum MJA, Vermeulen AM, Brokx JPL, van den Broek P. The relation between age at the time of cochlear implantation and long-term speech perception abilities in congenitally deaf subjects. Int J Pediatr Otorhinolaryngol 1997; 41:121-31.

7. Waltzman SB. Variables affecting speech perception in children. In: Waltzman SB, Cohen NL, eds. Cochlear implants. New York, NY: Thieme, 2000:199-224.

8. Ruben RJ. A time frame of critical/sensitive periods of language development. Acta Otolaryngol (Stockh) 1997;117: 202-5.
9. Osberger MJ, Fisher L, Zimmerman-Phillips S, Geier L, Barker MJ. Speech recognition performance of older children with cochlear implants. Am J Otol 1998;19:152-7.

10. Dowell RC, Blamey PJ, Clark GM. Factors affecting outcomes in children with cochlear implants. In: Clark GM, ed. Cochlear implants: XVI World Congress of Otorhinolaryngology-Head and Neck Surgery. San Diego, Calif: Singular Publishing Group, 1997:205-22.

11. Bench J, Bamford JM, Wilson IM, Cliff L. A comparison of the BKB sentence lists for children with other speech audiometry tests. Austral J Audiol 1979;1:61-6.

12. Skinner MW, Clark GM, Whitford LA, et al. Evaluation of a new spectral peak coding strategy for the Nucleus 22 channel cochlear implant system. Am J Otol 1994;15(suppl 2):1527.

13. Dunn LM, Dunn LM. Peabody Picture Vocabulary Test. 3rd ed. Circle Pines, Minn: American Guidance Service, 1997.

14. Blamey P, Sarant J, Serry T, et al. Speech perception and spoken language in children with impaired hearing. In: Proceedings of the Fifth International Conference on Spoken Language Processing, Sydney, Australia, 1998;6:2615-8. 


\section{University Library}

\section{- M M N E R VA A gateway to Melbourne's research publications}

Minerva Access is the Institutional Repository of The University of Melbourne

Author/s:

Dowell, RC;Dettman, SJ;Hill, K;Winton, E;Barker, EJ;Clark, GM

Title:

Speech perception outcomes in older children who use multichannel cochlear implants:

Older is not always poorer

Date:

2002-05-01

\section{Citation:}

Dowell, R. C., Dettman, S. J., Hill, K., Winton, E., Barker, E. J. \& Clark, G. M. (2002). Speech perception outcomes in older children who use multichannel cochlear implants: Older is not always poorer. ANNALS OF OTOLOGY RHINOLOGY AND LARYNGOLOGY, 111 (5), pp.97-101. https://doi.org/10.1177/00034894021110S520.

Persistent Link:

http://hdl.handle.net/11343/27600 